\title{
CASO 5-2014: Urticaria Grónica Idiopática o Urticaria espontánea y Omalizumab. Revisión del tema a propósito de un caso
}

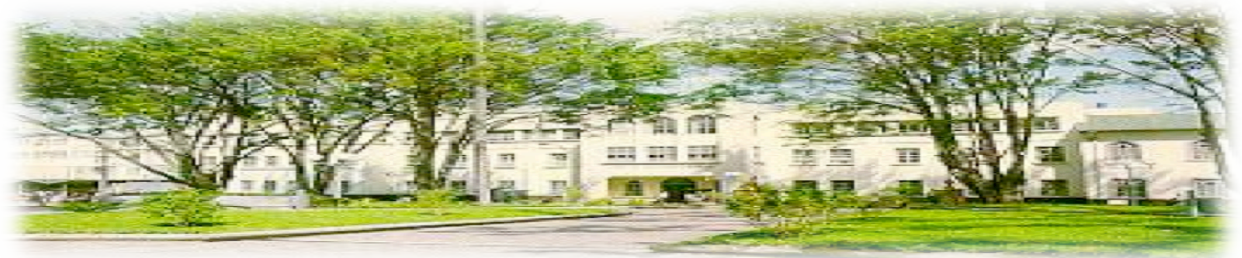

Haspital San quan de Dios. San Yosé. Costa Rica. Fundado en 1845

\section{Reporte de Gaso}

$\begin{array}{ll}\text { Recibido: } & 12 / 12 / 2013 \\ \text { Aceptado: } & 24 / 04 / 2014\end{array}$

${ }^{1}$ Médico Especialista en Medicina Interna y Alergología. Asistente del Servicio de Medicina Interna. Sección de Medicina, Hospital San Juan de Dios

${ }^{2}$ Estudiante de Medicina. Universidad de Costa Rica.

\section{RESUMEN}

La urticaria crónica idiopática (UCI), también conocida como urticaria crónica espontánea (UCE), es una enfermedad debilitante de la piel caracterizada por la presencia de lesiones intensamente pruriginosas (roncha o habones) que ocurren intermitente o continuamente por más de 6 semanas, con o sin angioedema, y donde no existe un desencadenante externo. Los antihistamínicos H1 no sedantes son la base actual de tratamiento y son los únicos agentes autorizados para su uso, estos son eficaces en $45 \%$ - $60 \%$ de los casos. Los tratamientos de segunda línea convencionales no tienen evidencia científica a favor o sus efectos adversos los hacen poco útiles. Presentamos el primer caso en Costa Rica de una paciente con UCI severa sin respuesta a anti histamínicos manejada con omalizumab, un anticuerpo monoclonal contra la IgE, la cual mostró una excelente respuesta, se hace una revisión de la evidencia a favor de este medicamento en pacientes refractarios al tratamiento convencional.

\section{PALABRAS CLAVE}

Urticaria Crónica Idiopática. Angioedema. Antihistamínicos. Omalizumab

\section{ABSTRACT}

Chronic idiopathic urticaria (ICU), also known as chronic spontaneous urticaria (CEU), is a debilitating disease of the skin characterized by intensely pruritic lesions (rash or hives) that occur intermittently or continuously for more than six weeks, with or without angioedema, and where there is no external trigger. H1 nonsedating antihistamines are the current basis 
for treatment and are the only agents approved for use that kind of patients these are effective in $45 \%-60 \%$ of cases. The conventional secondline treatments have no scientific evidence or adverse effects make them less useful. We report the first case in Costa Rica of a patient with severe UCI that was refractary to anti histamines, she was handled with omalizumab, a monoclonal antibody against $\operatorname{IgE}$, which showed an excellent response, we review the evidence in support of this drug

\section{KEY WORDS}

Chronic Idiopathic Urticaria. Angioedema. Antihistamines. Omalizumab.

\section{CASO CLÍNICO}

Paciente femenina de 40 años de edad, sin antecedentes patológicos de importancia. Consultó por cuadro de 4 semanas de urticaria, síntomas diarios de severidad 9 de 10 según la paciente, con afectación importante del sueño y desempeño diario, síntomas no asociados a una infección previa o a toma de medicamentos, no relación con alimentos. Se le prescribió ceterizina $10 \mathrm{mg}$ VO cada doce horas, ketotifeno $5 \mathrm{mg}$ cada doce horas y deflazacort $30 \mathrm{mg}$ VO cada día por siete días. A los 15 días la paciente no reportó mejoría de los síntomas por lo que se aumentó la dosis de Rupatadina a $20 \mathrm{mg}$ cada doce horas y montelukast $10 \mathrm{mg}$ VO cada día. En cita control a los 15 días sin mejoría, refierió que tres días antes de consultar tuvo que visitar el servicio de emergencias, donde le colocaron dexametasona y clorfenhidramina por vía parenteral, se agregó deflazacort, nuevo ciclo de siete días.

A la semana siguiente consultó nuevamente al servicio de emergencias, donde se le colocó nuevo esquema de dexametasona y clorfenhidramina parenterales, se mantuvo con el tratamiento indicado por tres semanas más sin mejoría.

A las 12 semanas de síntomas se realizó exámenes de laboratorio que mostraron hemograma, VES, PCR, complemento, frotis de heces y pruebas de función respiratoria normales,
IgE total en $69 \mathrm{UI} / \mathrm{ml}$, IgE por alimentos negativo. Se envió una muestra de sangre a Estados Unidos para medir anticuerpos contra IgE, el cual fue negativo. A las 14 semanas de síntomas se decidió, en vista de la no respuesta a las medidas convencionales utilizar ciclosporina, pero la paciente rechazó la opción debido a los posibles efectos adversos y riesgos asociados a este medicamento, por lo que se decidió utilizar omalizumab $150 \mathrm{mg}$ cada mes por tres meses.

La paciente se mantuvo con síntomas de igual intensidad durante la primera semana, en la visita de la segunda semana reportó una mejoría de intensidad de los síntomas de 4/10, dejando de afectar el sueño y su desempeño diario. Actualmente se encuentra en el sexto mes sin omalizumab y se mantiene con síntomas leves una o dos veces al mes, tratamiento actual rupatadina $10 \mathrm{mg}$ vo cada doce horas y ketotifeno cada doce horas.

En vista de la excelente evolución y de que es la primera vez que se utiliza omalizumab en nuestro país, tanto a nivel privado como en seguridad social para el manejo de urticaria espontánea, se decidió hacer una revisión sobre este tema.

\section{DISCUSIÓN}

\section{Definición y epidemiología}

La urticaria crónica idiopática (UCI), también conocida como urticaria crónica espontánea (UCE), es una enfermedad debilitante de la piel caracterizada por la presencia de lesiones intensamente pruriginosas (ronchas o habones) que ocurren intermitente o continuamente por más de 6 semanas $^{(1,2)}$ con o sin angioedema $\mathrm{y}$ donde no existe un desencadenante externo ${ }^{(3,4)}$.

La UCI afecta al $0.5 \%$ a $1 \%$ de los individuos (prevalencia de vida) $^{(5)}$ y puede ser altamente incapacitante $^{(2)}$. Generalmente tiene una duración prolongada de 1 a 5 años, pero puede persistir durante más de 5 años en el 11 al $14 \%$ de pacientes $^{(6)}$. Hasta $40 \%$ de los pacientes con urticaria por más de 6 meses tendrán urticaria 10 años más tarde y el $20 \%$ tendrá aún 20 años después $^{(5)}$. Sólo en Europa, más de 5 millones de 
pacientes se cree que sufren de síntomas de urticaria persistente ${ }^{(7)}$.

Muchos de los pacientes con urticaria crónica (UC) refieren una disminución considerable de la calidad de vida como consecuencia del prurito $^{(8)}$, con efectos negativos sobre el sueño, actividades diarias escuela $o$ trabajo $e$ interacciones sociales $^{(7)}$. El deterioro que acompaña a este trastorno ha sido comparado con el observado por los pacientes con enfermedades cardíacas ${ }^{(3,9)}$. Se cree que la UC tiene mayor impacto en calidad de vida que cualquier enfermedad alérgica $^{(1)}$.

\section{Fisiopatología}

La liberación de histamina de los mastocitos cutáneos ha sido asociada con la patogénesis de la urticaria aguda, mientras que en los pacientes con UC, los basófilos y la $\operatorname{IgE}$ también pueden desempeñar un papel importante ${ }^{(3,10)}$.

La principal célula efectora en la génesis del habón es el mastocito cutáneo, que tras su activación presenta un fenómeno de degranulación, liberación de histamina y de otros mediadores vasoactivos y proinflamatorios ${ }^{(9)}$, los opioides, la $\operatorname{IgE}$ (el mastocito tiene en su membrana un receptor de alta afinidad para $\operatorname{IgE}$ FceR1), los factores del complemento (C5A) o la sustancia $\mathrm{P}$, entre otros, son potenciales activadores del mastocito ${ }^{(8)}$. Una vez activado, el mastocito libera gránulos que contienen principalmente histamina y otros mediadores de la inflamación, como el factor activador de plaquetas (PAF), TNF alfa, IL-3, IL-4, IL-5, IL-6, IL-8, IL-13, GM-CSF, PGD-2 y leucotrienos (LTC4, LTD4, LTEA) ${ }^{(11)}$. La histamina, TNF alfa e IL-8 estimulan también a las moléculas de adhesión endotelial, con lo que se favorece la migración de eosinófilos, monocitos y neutrófilos desde el torrente sanguíneo a la piel ${ }^{(8)}$.

El 35-50\% de los pacientes tienen un mecanismo autoinmune, con autoanticuerpos frente a la cadena $\alpha$ del receptor de alta afinidad de $\operatorname{IgE}$ o contra la propia $\operatorname{IgE}^{(1,12)}$. Pacientes con UCI exhiben en promedio niveles de IgE total más altos en comparación con los controles $\operatorname{sanos}^{(13,14)}$.
En aproximadamente el $45 \%$ de los casos de UCI es posible detectar signos de autoinmunidad, por medio de las pruebas en piel mediante el uso de suero autólogo, utilizando el plasma del paciente, o mediante la detección de autoanticuerpos frente al receptor $\operatorname{IgE}$ de alta afinidad (FceR1), en los basófilos y mastocitos ${ }^{(15)}$. Sin embargo, sólo en algunos de estos pacientes puede demostrarse la presencia de autoanticuerpos contra el FceR1 o contra la propia $\operatorname{IgE}^{(15)}$.

En más de $50 \%$ de pacientes con UCI puede detectarse niveles elevados de anticuerpos IgE contra tiroperoxidasa (TPO). Estos autoanticuerpos IgE anti-TPO, activan la superficie del mastocito y podrían causar una degranulación autoalérgica del mastocito. El tratamiento con moléculas anti-IgE podría dar lugar a la rápida reducción de la IgE y esto por lo tanto a la supresión de los síntomas de urticaria $^{(11)}$

\section{Tratamiento tradicional}

Los antihistamínicos $\mathrm{H} 1$ no sedantes son la base actual para el tratamiento y son los únicos agentes autorizados para su uso en pacientes con $\mathrm{UCI}^{(3)}$. La dosis debe ser aumentada hasta cuatro veces la dosis estándar ${ }^{(7)}$. Este procedimiento es apoyado por los estudios actuales que muestran que dosis más altas son seguras y la respuesta es superior a la dosis estándar ${ }^{(16,17)}$. Los antihistamínicos son eficaces en el tratamiento de 45 al $60 \%$ de los pacientes; el resto son refractarios y logran poco o ningún beneficio incluso a dosis máximas ${ }^{(18)}$.

Las opciones de tratamiento para los pacientes que no tienen una respuesta a los antihistamínicos H1 incluye el uso de antihistamínicos $\mathrm{H} 2$, antagonistas de los receptores de leucotrienos, glucocorticoides sistémicos, ciclosporina, hidroxicloroquina, dapsona, metotrexato, sulfasalazina y gammaglobulina intravenosa. Ninguno de estos agentes todavía ha recibido aprobación para el tratamiento de la urticaria crónica idiopática. Además, los datos que apoyan el uso de estos medicamentos son limitados y a largo plazo el uso de algunos de estos agentes poseen efectos adversos importantes ${ }^{(19)}$. 
Las directrices actuales según la Administración de Medicamentos y Alimentos de los Estados Unidos (FDA por sus siglas en inglés) recomiendan un enfoque paso a paso en UCI, inicialmente, con el uso de antihistamínicos $\mathrm{H} 1$ no sedantes. Como segunda línea la terapia incluye la suplementación con bloqueadores H2 y modificadores de leucotrienos. Los inmunomoduladores (ciclosporina, mofetil, tacrolimus, hidroxicloroquina y sulfasalazina) se utilizan como agentes de tercera línea ${ }^{(20,21)}$. La ciclosporina es un tratamiento de tercera línea que cuenta con una recomendación basada en evidencia $^{(22)}$. Es eficaz pero tiene el potencial de efectos secundarios graves, necesitando monitoreo de la presión arterial y la función renal. La mayoría de las alternativas restantes se basan en la evidencia clínica de baja calidad ${ }^{(23)}$.

Los corticosteroides orales se pueden usar para tratar las exacerbaciones $\mathrm{UCI} / \mathrm{UCE}^{(24)}$; pero no se recomienda como una opción de tratamiento a largo plazo, dada la gran posibilidad de efectos secundarios asociados con su uso crónico ${ }^{(18)}$.

En la Cuarta Reunión del Consenso para la Actualización de las Guías de la Urticaria Crónica Espontánea realizada el mes de noviembre de 2012 en Berlín, se introdujo algunos cambios en el algoritmo de manejo de la urticaria crónica. Dado que en el algoritmo sólo se incluyen aquellas opciones terapéuticas avaladas con un grado de recomendación fuerte, se retiró del mismo el empleo de dapsona así como de los antihistamínicos-H2. Ante el fracaso del empleo de antihistamínicos-H1 no sedantes a dosis más altas, se recomienda su aumento hasta un máximo de cuatro veces la dosis usual ${ }^{(19)}$. Se recomienda como segunda línea de tratamiento el empleo de omalizumab, ciclosporina A o antileucotrienos ${ }^{(19)}$. Estos fármacos se añaden al empleo de los antihistamínicos- $\mathrm{H} 1^{(8)}$.

\section{Omalizumab}

El omalizumab es un anticuerpo monoclonal humanizado recombinante contra la $\mathrm{IgE}$, específicamente contra el dominio C3 de IgE, cerca el sitio de unión a los receptores de alta afinidad (FceRI) y baja afinidad (FceRII) en basófilos y mastocitos. Puede unirse a la $\operatorname{IgE}$ que está libre en suero o en el intersticio, pero no a las moléculas de IgE unidas a la superficie de la célula, ya que en este caso el sitio de unión a omalizumab está ocupado por el receptor ${ }^{(25)}$.

Es obtenido mediante la tecnología de ADN recombinante. Está compuesto por una fracción murina del 5\% y una fracción humana del 95\%. La fracción murina es la activa y está minimizada para evitar respuestas de anafilaxia. ${ }^{9}$

Fue aprobado en los Estados Unidos en el año 2003, para el tratamiento de asma grave o moderada, en pacientes mayores de 12 años y en Europa en 2005 para el tratamiento de asma grave en pacientes de 6 años en adelante ${ }^{(26)}$. Se recomienda 16 semanas de tratamiento para el tratamiento de asma grave ${ }^{(27)}$.

En los últimos años, se ha visto omalizumab con un uso más allá del ámbito de asma $^{(1)}$. Ha demostrado ser muy eficaz en la mejoría de los síntomas de la $\mathrm{UC}^{(22)}$. El fármaco también se ha utilizado en el tratamiento de rinitis alérgica, dermatitis atópica, mastocitosis y alergia a alimentos, así como un adyuvante en la inmunoterapia con alergenos y la urticaria ${ }^{(24)}$.

\section{Mecanismo de acción}

Se ha observado una reducción de la IgE libre en plasma a menos del $10 \%$ del valor del nivel basal $^{(13)}$ ya que se une a la molécula de IgE en la misma región que su receptor, en las células diana (basófilos y mastocitos), ésto es independientemente de su especificidad. En el caso de los receptores de alta afinidad presentes en basófilos y mastocitos, bloquea la unión IgEFceR1 e impide la activación celular y la liberación de sustancias vasoactivas, como la histamina y otros mediadores inflamatorios como leucotrienos, triptasa, quimasa, prostaglandina D2 y citocinas $^{(9)}$.

La IgE puede modular el grado de expresión de sus propios receptores de alta y baja afinidad ${ }^{(28)}$. La ocupación por IgE del FceR1 de los mastocitos y basófilos determina los niveles de expresión del receptor en superficie, de manera que a mayor concentración de $\operatorname{IgE}$, aumenta la densidad de sus receptores y la reactividad de los mastocitos y basófilos, que disminuyen con la reducción de sus concentraciones ${ }^{(8)}$. 
La rápida mejoría de los síntomas de urticaria reportada en casos anteriores tratados con omalizumab, indica que la IgE juega un papel importante en la UC. Sin embargo, aunque los mecanismos anti-IgE se cree que son el principal modo de acción para el omalizumab, se debe tomar en cuenta el papel de otros mecanismos tales como la inducción de la apoptosis de eosinófilos, regulación a la baja de las citocinas inflamatorias IL-2, IL-4, IL-13 y TNF- $\alpha$ y el aumento en la actividad de las células CD4 por liberación de ATP. Estas observaciones ofrecen una explicación de los buenos resultados también en los pacientes con bajos niveles de $\operatorname{IgE}{ }^{(23)}$.

La reducción en el número de receptores de alta afinidad podría explicar la eficacia del omalizumab en el tratamiento de la UCI con anticuerpos contra dicho receptor, ya que disminuiría su diana. Sin embargo, existen datos clínicos y de laboratorio que sugieren que el mecanismo del omalizumab es multifactorial y que además podría actuar sobre otras dianas celulares en el sistema inmunitario aún no bien estudiadas $^{(9,24)}$.

El omalizumab disminuye el número de autoanticuerpos IgE antitiroperoxidasa e inhibe la degranulación del mastocito al reducir la densidad de receptores de IgE en su superficie ${ }^{(8)}$, la activación de los linfocitos B, TNF-alfa y de IL4, además produce un aumento de la síntesis de IFN-gamma en el suero de pacientes con $\mathrm{UCI}^{(9)}$.

\section{Dosis}

El omalizumab está disponible en jeringas precargadas de aplicación subcutánea. Existen 2 presentaciones: 75 y $150 \mathrm{mg}$. La dosis apropiada y la frecuencia de administración de omalizumab se determinan a partir de la concentración basal de $\operatorname{IgE}(\mathrm{UI} / \mathrm{ml})$, medida antes de iniciar el tratamiento y del peso corporal $(\mathrm{kg})$ del paciente $^{(2)}$. Siendo la dosis aprobada del fármaco para el tratamiento del asma de $0.016 \mathrm{mg} / \mathrm{kg}$ por $\mathrm{UI} / \mathrm{ml}$ de $\mathrm{IgE}$, teniendo en cuenta que la cuantificación de IgE inicial no debe ser superior a $1.500 \mathrm{UI} / \mathrm{ml}^{(9)}$.

La dosis única de omalizumab se absorbe lentamente, alcanzando concentraciones máximas después de una media de 7 a 8 días, lo que se atribuye a que el medicamento podría tener un efecto directo sobre los mastocitos y no sólo sobre la $\operatorname{IgE}{ }^{(8,27)}$.

Se suele repetir la administración a dosis de 2 o 4 semanas hasta las 16 semanas del inicio. Con la dosis recomendada la $\operatorname{IgE}$ sérica decrece rápidamente y alcanza un nadir de $<50 \mathrm{ng} / \mathrm{ml}$ (20.8 UI/ml), niveles a los que se ha demostrado beneficios en el asma alérgica y en la rinitis y los efectos pueden durar hasta meses si se consigue bloquear toda la IgE sérica ${ }^{(29)}$.

Respecto a su eliminación, el omalizumab tiene una vida media de 26 días, con un aclaramiento promedio de $2.4 \pm 1.1 \mathrm{ml} / \mathrm{kg} /$ día por parte del sistema reticuloendotelial. Se ha observado que el efecto del omalizumab se mantiene durante meses. No es necesario realizar un ajuste de dosis en función de la edad (12-76 años), raza, grupo étnico o $\operatorname{sexo}{ }^{(9)}$.

El omalizumab en dosis de $150 \mathrm{mg}$ y $300 \mathrm{mg}$ mejoró significativamente los resultados en pacientes con UCI sintomáticos, a pesar del uso de antihistamínicos H1 a la dosis aprobada ${ }^{(13,30,31)}$. Maurer y colaboradores realizaron un estudio con 323 adultos que no respondieron a tratamiento a pesar de las dosis recomendadas de anti histaminicos, casi $75 \%$ de los pacientes eran mujeres, edad media 45 años, se asignaron tres grupos con dosis de 75, 150 y $300 \mathrm{mg}$, en tres dosis subcutáneas dadas cada 4 semanas, el grupo control recibió placebo, todos los pacientes fueron manejados por 16 semanas. Los pacientes presentaban una escala de prurito promedio (de 0-21 puntos) de 14 puntos. Las dosis más altas de omalizumab se asociaron con mejores reducciones del prurito. A las doce semanas, la reducción promedio del prurito fue de 5.1 puntos con placebo, 5.9 puntos con omalizumab $75 \mathrm{mg}, 8.1 \mathrm{con}$ omalizumab $150 \mathrm{mg}$ y 9.8 con $300 \mathrm{mg}$. Las tasas de efectos adversos fueron similares a placebo $^{(3)}$, estos datos coinciden con estudios previos ${ }^{(27)}$.

Ivyanskiy y colabodores analizaron 19 pacientes con UC resistente al tratamiento, elegidos por el Departamento de Dermatología del Hospital Bispebjerg en Copenhague. Los pacientes fueron tratados con una una dosis de $150 \mathrm{mg}$ de omalizumab cada dos semanas y se encontró que 
un total del $84 \%$ experimentó resolución de los síntomas en un grado que excedió el efecto de los tratamientos anteriores, el efecto sintomático del tratamiento se produjo en muchos de los pacientes después de uno o unos pocos días y no se informaron efectos secundarios graves ${ }^{(32)}$.

La evidencia a favor del omalizumab en el manejo de la UCI refractaria es cada vez mayor. Existe disminuciones estadísticamente significativas de la urticaria, además de una completa protección contra la aparición de ronchas en el $70.4 \%$ frente a $4.5 \%$ de los pacientes tratados con placebo ${ }^{(13)}$, otro estudio mostró respuestas completas en 23 y $53 \%$ de los pacientes con 150 y $300 \quad \mathrm{mg}$, respectivamente $^{(13,22)}$, se concluye que la dosis fija de omalizumab, independiente del nivel de IgE o peso, es eficaz y suficiente para el manejo de los pacientes con $\mathrm{UC}^{(1)}$.

\section{CONCLUSIONES}

La Urticaria Crónica Idiopática es una entidad poco frecuente, con respuesta variable a los tratamientos convencionales y que genera gran impacto en la calidad de vída de los pacientes que la sufren, llevando incluso a problemas de sueño

El tratamiento recomendado es escalonado y basado en ahtihistamínicos. Según la evolución se recomienda agregar otros esquemas incluyendo inmunomoduladores.

El omalizumab es una opción de tratamiento cososo y no aprobado por la FDA para esta indicación, no es una opción viable para todos los pacientes UCI, sin embargo, puede a la largo plazo ayudar a sufragar los costos de atención de salud para pacientes con CU refractaria.

\section{BIBLIOGRAFÍA}

1. Viswanathan $\mathrm{R}$ Moss $\mathrm{M}$ Mathur $\mathrm{S}$. Retrospective analysis of the efficacy of omalizumab in chronic refractory urticaria. Allergy Asthma Proc 2013;34:446-452.

2. Armengot M Rodrigo M Pont B Quecedo V Gimeno E. Omalizumab in chronic urticaria: a retrospective series of 15 cases. Dermatologic Therapy 2013;26:257-259.

3. Maurer $\mathrm{M}$ Rosen $\mathrm{K}$ Hsieh $\mathrm{HJ}$ et al. Omalizumab for the treatment of chronic idiopathic or spontaneous urticaria. N Engl J Med 2013;368:924-935.

4. Groffik A Mitzel-Kaoukhov H Magerl M Maurer M Staubach P. Omalizumab-an effective and safe treatment of therapyresistant chronic spontaneous urticaria. Allergy 2011;66:303-305.

5. Le Fourn E Chosidow B Doutre O Lorette M. Study Design and Quality of Reporting of Randomized Controlled Trials of Chronic Idiopathic or Autoimmune Urticaria: Review. PLOS ONE 2013;8:1-7.

6. Maurer M Rosen K Hsieh H. Omalizumab for chronic urticaria. $\mathrm{N}$ Engl J Med 2013;368:2530.

7. Metz M Maurer M. Omalizumab in chronic urticaria. Curr Opin Allergy Clin Immunol 2012;12:406-411.

8. Curto-Barredo L Silvestre J Giménez-Arnau A. Actualización en el tratamiento de la urticaria crónica. Actas Dermosifiliográficas 2013;826:1-14.

9. Frances L Leiva-Salinas M Silvestre J. Omalizumab in the Treatment of Chronic Urticaria. Actas Dermosifiliogr. 2013. http://dx.doi.org/10.1016/j.ad.2013.06.002

10. Caffarelli C Cuomo B Cardinale F. Aetiological factors associated with chronic urticaria in children: a systematic review. Acta Derm Venereol 2013;93:268-272.

11. Metz M Krull C Maurer M. Histamine, TNF, C5a, IL-6, -9, -18, -31, -33, TSLP, Neopterin, and VEGF are not elevated in chronic spontaneous urticaria. J Dermatol Sci 2013;70:222-225.

12. Posthumus J Tinana A Mozena JD Steinke J Borish L. Autoimmune mechanisms in chronic idiopathic urticaria. J Allergy Clin Immunol 2012;130:814-816 e4.

13. Metz M Ohanyan T Church MK Maurer M. Omalizumab is an effective and rapidly acting therapy in difficult-to-treat chronic urticaria: A retrospective clinical analysis. J Dermatol Sci 2013. En prensa.

14. Popov T. Challenges in the management of chronic urticaria. World Allergy Organ J 2011;4:S28-31.

15. Konstantinou GN Asero R Ferrer $\mathrm{M}$ et al. EAACI taskforce position paper: evidence 
for autoimmune urticaria and proposal for defining diagnostic criteria. Allergy 2013;68:27-36.

16. Krause K Spohr A Zuberbier T Church MK Maurer M. Up-dosing with bilastine results in improved effectiveness in cold contact urticaria. Allergy 2013;68:921-928.

17. Maurer M Magerl M Metz M Zuberbier T. Revisions to the international guidelines on the diagnosis and therapy of chronic urticaria. J Dtsch Dermatol Ges 2013. En prensa

18. Kaplan A Ashby D Canvin M Zazzali J Conner J. Omalizumab in patients with symptomatic chronicidiopathic/spontaneous urticaria despite standard combination therapy. Journal of Allergy and Clinical Immunology 2013;132:101-109.

19. Maurer M Magerl M Metz M Zuberbier T. Diagnosis and therapy of chronic urticariawhat is expected from the revision and update of the international guidelines? A report of the public consensus conference “URTICARIA 2012”. Hautarzt 2013;64:638643.

20. Kropfl L Maurer M Zuberbier T. Treatment strategies in urticaria. Expert Opin Pharmacother 2010;11:1445-1450.

21. Fernando S Broadfoot A. Chronic urticaria-assessment and treatment. Aust Fam Physician 2010;39:135-138.

22. Labrador M Velasco A Jauregui M Sastre I Barta J. Efficacy of omalizumab in chronic spontaneous urticaria refractory to conventional therapy: analysis of 110 patients in real-life practice. Expert Opin Biol Ther 2013;13:1225-1228.

23. Kaplan A Ledford D Ashby M. Omalizumab in patients with symptomatic chronic idiopathic/spontaneous urticaria despite standard combination therapy. J Allergy Clin Immunol 2013;132:101-109.

24. Iemoli E Piconi S Fusi A Borgonovo L Borelli M Trabattoni D. Immunological effects of omalizumab in chronic urticaria: a case report. J Investig Allergol Clin Immunol 2010;20:252-254.

25. Bergstrom K. Chronic urticaria: omalizumab and review of therapeutic options. J Drugs Dermatol 2013;12:715-716.

26. Metz M Altrichter S Ardelean E et al. Antiimmunoglobulin E treatment of patients with recalcitrant physical urticaria. Int Arch Allergy Immunol 2011;154:177-180.

27. Saini S Rosen K Hsieh H. A randomized, placebo-controlled, dose-ranging study of single-dose omalizumab in patients with H1antihistamine-refractory chronic idiopathic urticaria. J Allergy Clin Immunol 2011;128:567-573 e1.

28. Dreyfus DH. Observations on the mechanism of omalizumab as a steroidsparing agent in autoimmune or chronic idiopathic urticaria and angioedema. Ann Allergy Asthma Immunol 2008;100:624-625.

29. Labrador-Horrillo M Valero A Velasco M et al. Efficacy of omalizumab in chronic spontaneous urticaria refractory to conventional therapy: analysis of 110 patients in real-life practice. Expert Opin Biol Ther 2013;13:1225-1228.

30. Lefevre AC Deleuran M Vestergaard C. A long term case series study of the effect of omalizumab on chronic spontaneous urticaria. Ann Dermatol 2013;25:242-245.

31. Kaplan AP Joseph K Maykut RJ Geba GP Zeldin RK. Treatment of chronic autoimmune urticaria with omalizumab. J Allergy Clin Immunol 2008;122:569-573.

32. Ivyanskiy I Sand C Thomsen S. Omalizumab for chronic urticaria: a case series and overview of the literature. Case Rep Dermatol 2012;4:19-26.

\section{CONFLICTO DE INTERÉS}

Los autores declaran que no existe ningún conflicto de interés en el presente reporte. 